\title{
Glycidyl-Methacrylate-Based Electrospun Mats and Catalytic Silver Nanoparticles
}

\author{
Mustafa M. Demir,* Gökçe Uğur, Mehmet Ali Gülgün, Yusuf Z. Menceloğlu
}

P(AN-GMA) and PGMA fibers coated with monodisperse silver nanoparticles have been prepared by a combination of electrospinning and electroless plating. The morphology of the electrospun fibers remains unchanged after surface hydrazination. Oxidation of hydrazine in an ammoniacal solution of $\mathrm{AgNO}_{3}$ reduces and deposits silver atoms along the fiber surface, which then coalesce to Ag particles. The size of the silver nanoparticles is varied between 20$60 \mathrm{~nm}$. Since the density of the active sites for silver reduction is lower in P(AN-GMA), a smaller particle size could be obtained. The catalytic activity of the silver nanoparticles has been confirmed.

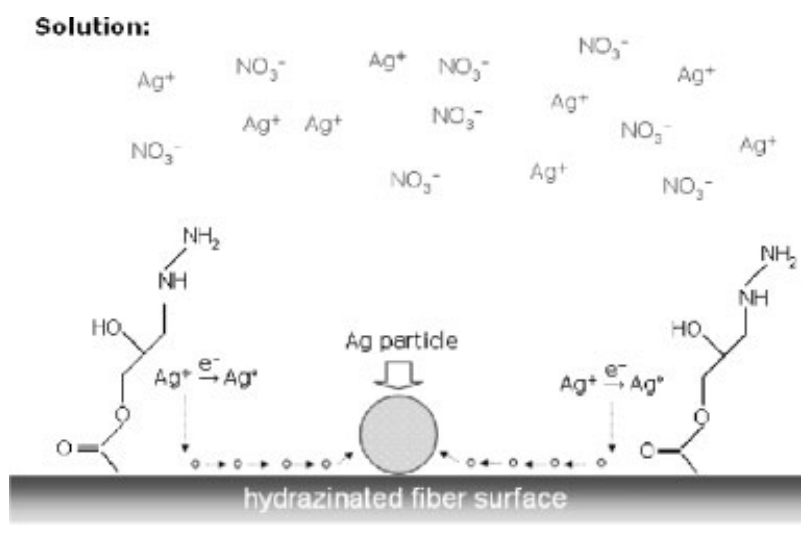

\section{Introduction}

Metal nanoparticles possess unique, often size-dependent properties that are different to the properties in their respective bulk form. ${ }^{[1,2]}$ Among the other metals, silver is particularly attractive because of its significantly wide spread applications in optics, ${ }^{[3]}$ electronics, ${ }^{[4]}$ biology, ${ }^{[5]}$ and catalysis. ${ }^{[6]}$ In particular, its application in catalysis is important since it often combines the characteristics of high reactivity and selectivity. There are many reactions catalyzed by metallic silver such as oxidative conversion of methanol into formaldehyde, ${ }^{[7]}$ selective butadiene epoxidation, ${ }^{[8]}$ and oxidation of D-glucose. ${ }^{[6]}$ High efficiency in catalysis often requires a uniform size and a homogeneous

\section{M. Demir}

Chemistry Department, izmir Institute of Technology, Gülbahçe Köyü, Urla, 35430 Turkey

Fax: +90 232750 7509; E-mail: mdemir@iyte.edu.tr

G. Uğur, M. A. Gülgün, Y. Z. Menceloğlu

Faculty of Engineering and Natural Sciences, Sabancı University

Orhanlı 34956 istanbul Turkey distribution throughout a suitable substrate. Control over size of nanoparticles and of agglomeration are key points in the preparation of catalytic particles.

Nanoparticles are often surface modified by surfactant molecules to stabilize them against undesirable aggregation especially when they are associated with polymers. ${ }^{[9]}$ However, in most cases, surface modification by surfactant molecules leads to the loss of certain properties, like catalytic activities, compared to naked metal particles. ${ }^{[0]}$ A method that stabilizes the metal particles without sacrificing the catalytic activity is needed. Fabrication of nanoparticles either by reduction or by heat treatment of metal ions in situ appears to be the most efficient way for stabilization of the metal particles. ${ }^{[11]}$ Various metals like $\mathrm{Fe}^{[12]} \mathrm{Pd},{ }^{[13]} \mathrm{Cu}^{[14]} \mathrm{Au}^{[15]} \mathrm{Ag},{ }^{[16]}$ and $\mathrm{Ni}^{[17]}$ have been encapsulated employing the in-situ generation of particles in thin polymer films. Although the fabrication of nonaggregated metal nanoparticles can be obtained in situ without using surfactants, an efficient catalytic performance cannot be achieved because of the encapsulation of catalytic particles in a polymer matrix. The presence of a polymer layer surrounding the particles limits the 
interaction between reactive species and the particle surface. In this work, catalytic silver particles are selectively deposited onto the surface of a porous mat such that the particles are easily accessible to various chemical reagents. An electrospun mat is used as polymeric substrate with its high surface area and easily accessible structure.

Block copolymers have been widely employed as structure directing agents in the production of metal particles in polymer films. ${ }^{[18,19]}$ Microphase separation in block copolymers is a well-studied phenomenon to produce morphologies by phase separation. The ability to control the spatial and organizational morphologies of block copolymers makes these materials attractive for fabrication of nanostructures. The synthesis is based on the spontaneous phase separation of copolymer molecules which restricts metal species within nanometer-scale regions in the bulk morphology. Metal ions interact with one of the blocks, which assemble to produce nanometerscale domains that contain the metal ions for later reduction to zerovalent particles. The application of copolymer chemistry in the production of an electrospun mat can be an efficient approach to obtain a substrate system where metal particles remain non-aggregated. In our previous study, we have shown in-situ generation of Pd particles in/on electrospun fibers of statistical copolymers of acrylonitrile and acrylic acid with different compositions. ${ }^{[13]}$ The catalytic activity of electrospun fiber-supported Pd particles was 4.5 times higher than the current $\mathrm{Pd} / \mathrm{Al}_{2} \mathrm{O}_{3}$ catalyst in a hydrogenation reaction.

Electrospinning is a versatile fiber spinning technique that allows the production of continuous polymer fibers down to several nanometers under a uniform electrical field. ${ }^{[20-22]}$ In a typical procedure, a probe of a high voltage generator is immersed into a polymer solution. A high voltage on the order of several kilovolts is applied to the polymer solution that is filled into a narrow tube. Several centimeters away from its nozzle, a grounded conductive sheet is placed perpendicular to the pipette. When the applied electrical field overcomes the surface tension of the solution, a jet is created, which comes out from the nozzle to the grounded sheet. Upon evaporation of solvent, a thin polymeric mat is formed on the grounded sheet, which consists of randomly distributed fibers. Electrospinning provides a simple and fast approach to produce a fibrous polymeric mat.

Silver particles have been associated with many polymers using different pathways based on electrospinning. Recently, $\mathrm{Xu}$ et al. ${ }^{[23]}$ reported the preparation and antibacterial activity of silver particles in biodegradable electrospun poly(L-lactide) fibers for microorganism reduction. Silver particles are reduced in the nanofibers from $\mathrm{AgNO}_{3}$ at $80^{\circ} \mathrm{C}$ in an atmosphere of hydrogen at $0.8 \mathrm{MPa}$ for $48 \mathrm{~h}$. The antibacterial efficiency of the silver and the biodegradability of poly(L-lactide) are combined in the same material whose clinical applications as a wound dressing and anti-adhesion membrane were emphasized. ${ }^{[24,25]}$ Patel et al. ${ }^{[10]}$ presented the preparation of porous silica nanofibers that contain catalytic silver nanoparticles by sol-gel chemistry and an electrospinning technique. Silver nanoparticles were nucleated from $\mathrm{AgNO}_{3}$ at $200-800{ }^{\circ} \mathrm{C}$ for 30 to 150 min while the polymer precursor is calcinated. The catalytic activity of the silver incorporated into the fiber mats was assessed by a reduction of methylene blue dye. Another example using heat treatment for the nucleation of silver particles was shown by Jin et al. ${ }^{[26]}$ Large scale fabrication of the particles was performed using heat treatment of poly(vinyl pyrrolidone) $/ \mathrm{AgNO}_{3}$. In addition to the temperature-induced particle fabrication, the nucleation of particles on electrospun cellulose acetate fibers can be performed by UV radiation. ${ }^{[27]}$ A cellulose acetate solution that contained $\mathrm{AgNO}_{3}$ was subjected to electrospinning and the resulting electrospun mat was irradiated with UV light. The authors showed that silver particles nucleate in general at the surface of the fibers.

Contrasting from the literature, herein, catalytic silver nanoparticles were selectively deposited onto the surface of glycidyl methacrylate (GMA) based electrospun fibers by a redox reaction. The process takes place in an aqueous solution of $\mathrm{AgNO}_{3}$ at ambient conditions using electroless deposition. GMA as comonomer is rather useful, since the pendant oxirane ring can be opened and a range of functionalities can be introduced to the polymer backbone. ${ }^{[28-31]}$ Modification of the fiber surface with the reducing agent hydrazine provided binding sites for nucleation of metal atoms. Silver cations were reduced into metallic silver that diffused and coalesced into particles along the fiber's surface. Although many methods are available for coating, such as baking ${ }^{\left[{ }^{[32]}\right.}$ chemical vapor deposition, ${ }^{[33]}$ and ion-sputtering, ${ }^{[34]}$ the electroless plating is found to be very effective because of applicability on insulators and complex-shaped structures like electrospun mats. ${ }^{[35]}$ Electroless plating is especially suitable to silver plating since silver is a metal that facilitates the autocatalytic reaction for its reduction. ${ }^{[35]}$ Silver particles can be deposited evenly along edges, inside cavities, and cover irregularly shaped objects that are otherwise difficult to coat evenly. Control of particle sizes in the range of 20-60 $\mathrm{nm}$ was achieved by varying the GMA fraction of the copolymer and/or the deposition time.

\section{Experimental Part}

Acrylonitrile (AN) (Fluka), glycidyl methacrylate (GMA) (Fluka), methylene blue (Alrich), $\mathrm{NaBH}_{4}$ (Aldrich), and hydrazinium hydroxide (100\%; E. Merck) were of analytical grade and were 
used without any further purification. AN and GMA monomers were polymerized by radical polymerization in dimethylformamide (DMF) using 1 wt.-\% ammonium persulfate initiator. For the synthesis of the copolymer, polymerization was carried out for the mixture of AN and GMA (60:40) over a period of $24 \mathrm{~h}$ at $50^{\circ} \mathrm{C}$. The copolymer composition was estimated from the ${ }^{1} \mathrm{H}$ NMR spectra by integration of the characteristic peaks of oxirane over those of acrylonitrile.

Scheme 1 outlines the procedure for the fabrication of composite fibers. The details of that electrospinning process have been described elsewhere. ${ }^{[36]}$ Polymer solutions were subjected to an electrical field of $1.53 \mathrm{kV} \cdot \mathrm{cm}^{-1}$. Instrumental parameters (spinning distance: $8 \mathrm{~cm}$ and voltage: $12.2 \mathrm{kV}$ ) were kept constant for all experiments. An electrospun film that could be detached from the substrate surface was obtained in $6 \mathrm{~h}$. These thick polymer films were immersed into a dilute hydrazine solution overnight for surface modification. They were washed with excess distilled water twice to remove any free hydrazine molecules. For silver deposition on the fibers, the hydrazine-modified electrospun mats were introduced into a mixture of $5 \mathrm{~mL}$ of a $0.1 \mathrm{M} \mathrm{AgNO}_{3}$ solution, $0.5 \mathrm{~mL}$ of a $1 \mathrm{~m} \mathrm{KOH}$ solution, and $1 \mathrm{~mL}$ of concentrated $\mathrm{NH}_{3}$ solution in a closed glass bottle. The precipitation of the metal onto the nanofiber took place within a few minutes. The deposition time refers to the duration during which electrospun mats were subjected to the metal ion solution. It ranged from $1 \mathrm{~min}$ to $24 \mathrm{~h}$. Thermogravimetric analysis (TGA) and dynamic thermal analysis (DTA) were performed using a Netzch STA449C under an oxidative atmosphere. The heating rate was $20^{\circ} \mathrm{C}$ per minute from 27 to $1000^{\circ} \mathrm{C}$. The morphology of the fiber mats (fiber diameters, and the distribution and size of the silver particles) was investigated using both scanning electron microscopy (SEM, LEO Supra 35) and transmission electron microscopy (TEM, JEM 2000FX, JEOL). Particle size distributions were obtained from statistical treatment of SEM and TEM images by measuring the length of typically not less than 100 particles with the help of the software of the microscopes.

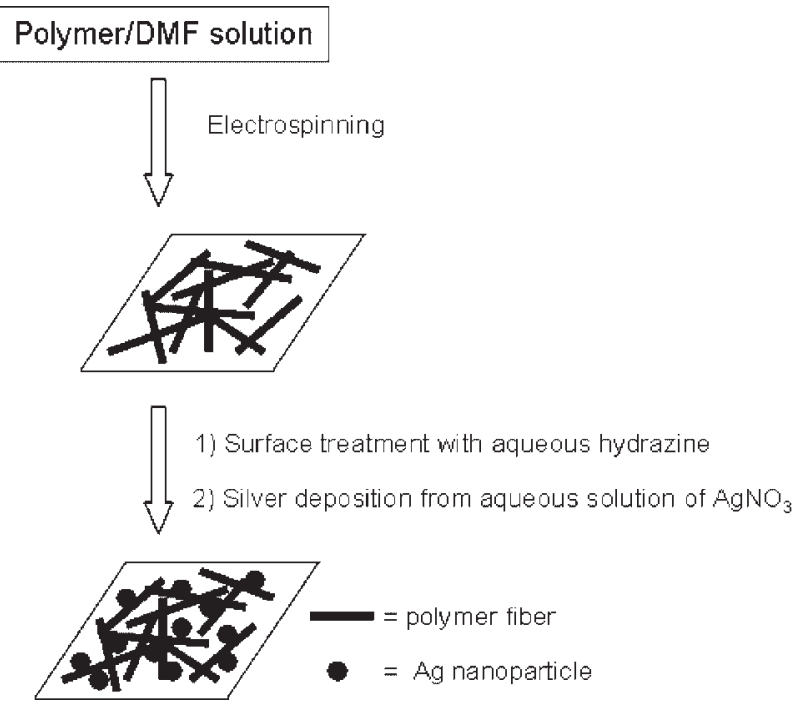

Scheme 1. Experimental steps for the synthesis of composite fibers based on silver particles and electrospun fibers.
The catalytic activity of the silver particles was analyzed employing a model redox reaction that takes place between methylene blue and $\mathrm{NaBH}_{4}$. An electrospun mat $(7 \mathrm{mg}$ consisting of 20 wt.-\% of silver) was immersed into a mixture of $3 \mathrm{~mL}$ of $0.02 \times 10^{-3} \mathrm{M}$ methylene blue and $1 \mathrm{~mL}$ of $0.1 \mathrm{M} \mathrm{NaBH}_{4}$ under magnetic stirring. The intensity of the blue color of the reaction mixture decreased and eventually turned colorless with time. The extent of the reaction was followed by absorption of the reaction mixture using optical spectroscopy (Varian Cary 50, $500-800 \mathrm{~cm}^{-1}$ ).

\section{Results and Discussion}

Electrospun fibers of P(AN-GMA) and PGMA were obtained from their solutions in DMF. The weight fractions of the polymer solutions are 0.30 and 0.45 for $\mathrm{P}(\mathrm{AN}-\mathrm{GMA})$ and PGMA, respectively. The corresponding diameters of the fibers fall in the range of 0.2-1.4 $\mu \mathrm{m}$. As an example of the spinnability of GMA-based polymers, electrospun fibers of a copolymer of AN and GMA (58: 42 in terms of mole ratio) are shown in Figure 1 . Submicrometer diameter P(AN-GMA) fibers were randomly deposited on an Al-foil substrate and formed a mat-like structure that has nano/ submicrometer scale porosity.

Silver nanoparticles were selectively generated on the surface of electrospun fibers by electroless plating. A two-step procedure was followed for the production of metal nanoparticles. In the first step, a reducing agent, hydrazine, was immobilized onto the fibers surface. Hydrazine is a strong nucleophile and attacks the highly strained three-membered oxirane ring of the glycidyl methacrylate residue. Hydrazination was revealed by IR spectroscopy through the disappearance of the asymmetric stretching bands of the oxirane group at $1129 \mathrm{~cm}^{-1}$ and the appearance of bands at $3216 \mathrm{~cm}^{-1}$, which

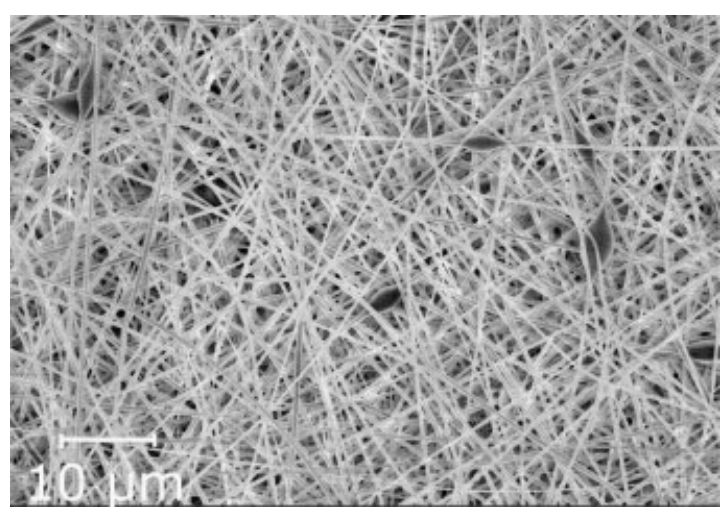

Figure 1. An overview SEM image of $\mathrm{P}(\mathrm{AN}-\mathrm{GMA})$ fibers electrospun from 30 wt.-\% solid content in DMF solution. The fibers have an average diameter of $0.17 \pm 0.05 \mu \mathrm{m}$ and an electrical field of $1.53 \mathrm{kV} \cdot \mathrm{cm}^{-1}$. The AN and GMA content in P(AN-GMA) is 58 and $42 \mathrm{~mol}-\%$, respectively. 


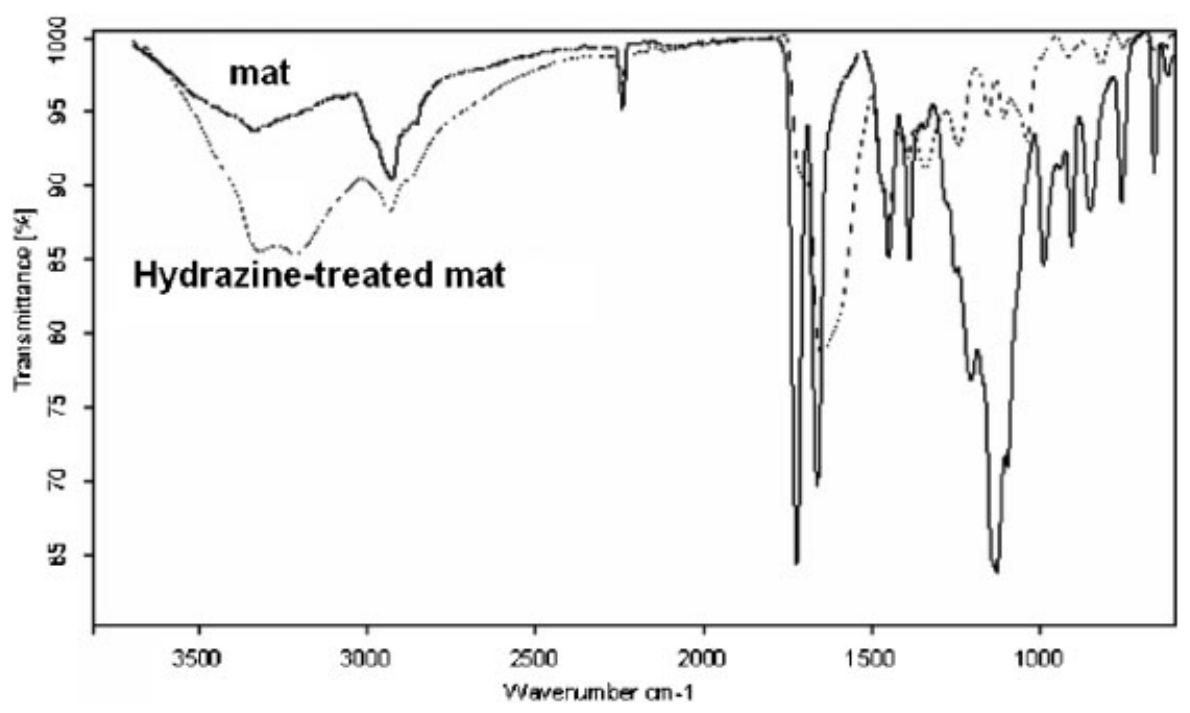

Figure 2. IR spectra of $\mathrm{P}(\mathrm{AN}-\mathrm{GMA})$ mats before and after hydrazine-treatment. The appearance of symmetric stretching of the primary amine at $3216 \mathrm{~cm}^{-1}$ and disappearance of the asymmetric stretching of the oxirane group at $1129 \mathrm{~cm}^{-1}$ indicates successful hydrazination of the fibers' surface.

originate from symmetric stretching of the $-\mathrm{NH}_{2}$ (primary amine) group ${ }^{[37]}$ (Figure 2). The morphology of the fibers remained unchanged after the surface modification. In the second step, the hydrazine-modified electrospun mat was introduced into an aqueous solution of $\mathrm{AgNO}_{3}$. Silver cations were reduced into metallic silver by hydrazine molecules that were immobilized on the fibers' surfaces. Consequently, nanometer-scale silver particles nucleated on the surface of the fibers. Hydrazinated-oxirane groups provided potential nucleation centers and the Ag particles were most likely nucleated at or near the hydrazinatedoxirane groups. The presence of Ag particles was imaged by SEM and TEM. Figure 3 illustrates electron micrographs of silver-coated electrospun fibers. Figure 3(a) shows a low magnification overall view of $\mathrm{P}(\mathrm{AN}-\mathrm{GMA}) / \mathrm{Ag}$ composite fibers. A large number of silver nanoparticles were formed and were evenly distributed on the fibers surface. Figure $3(b)$ provides a high magnification image of a $\mathrm{P}(\mathrm{AN}-\mathrm{GMA}) / \mathrm{Ag}$ fiber. The particles on the fiber surface are mostly separate islands. X-Ray diffraction was used to identify silver in this composite system. The diffraction peaks that correspond to the (111), (200), and (220) planes of silver were observed at $38.1,44.3$, and $64.4^{\circ}$, respectively. The reflections detected on the X-ray spectrum match those of metallic silver (JCPDS card no: 04-0783). The size of the Ag crystallites was estimated by peak broadening using the Debye-Scherrer formula. The average size of $\mathrm{Ag}$ crystallites obtained on the P(AN-GMA) fibers was $40 \pm 3 \mathrm{~nm}$. This dimension is very close to the particle diameter observed in the electron micrographs. This agreement indicates that silver particles nucleated on the fiber surface are single crystalline.
Silver nanoparticles were produced on both P(AN-GMA) and PGMA substrate fibers for different deposition times. The $\mathrm{P}(\mathrm{AN}-\mathrm{GMA})$ substrate is a statistical copolymer of AN and GMA where the comonomers are randomly distributed on the copolymer chain. The mole fraction of GMA, i.e., oxirane groups on the fibers' surfaces, is 0.42 . The other substrate is a homopolymer of GMA. Figure 4 illustrates particle diameters for different coating times on the two substrates: a) $\mathrm{P}(\mathrm{AN}-\mathrm{GMA})$ fibers, and b) PGMA fibers. In both cases, the particle size shows a linear increase with the logarithm of coating time. Initial particle sizes and particle growth rates on these two substrates were not identical because of the difference in the content of the oxirane group on the polymer chains. The particles nucleated on $\mathrm{P}(\mathrm{AN}-\mathrm{GMA})$ fibers were initially $\approx 10 \mathrm{~nm}$ smaller in diameter than the ones obtained on PGMA fibers. However, after $24 \mathrm{~h}$ of deposition time, mean diameters of the silver particles were $60 \mathrm{~nm}$ in both cases. This result suggests that the particles produced on $\mathrm{P}(\mathrm{AN}-\mathrm{GMA})$ grow faster than the ones nucleated on PGMA fibers, at least in the range of deposition time employed in this work. Apparently, particle-growth mechanisms on the two fibers seem different. Based on these preliminary results, two models were suggested considering the content of GMA residue on polymer backbones. It is likely that hydrazinated-oxirane groups facilitate and localize nucleation events of silver particles on the fibers' surfaces. Silver cations were reduced into metallic silver atoms on these reactive sites. After the electron exchange, metal atoms diffuse along the fiber surface and subsequently either nucleate new particles or adhere to an already existing nanoparticle to cause it to grow. In the case of PGMA, the fibers have nearly two times more oxirane groups compared to P(AN-GMA); therefore, the density of active sites for particle nucleation is higher on the PGMA fibers. A higher number of silver atoms were reduced on the surface of the electrospun fibers and formed larger silver particles at the same $\mathrm{AgNO}_{3}$ concentration and exposure time. This observation infers that particle growth is predominantly controlled by diffusion of the silver atoms on the surface the PGMA fibers. However, the mechanism of particle growth is somewhat different on $\mathrm{P}(\mathrm{AN}-\mathrm{GMA})$. The surface of the $\mathrm{P}$ (AN-GMA) fibers is nearly half as much populated by oxirane groups as the PGMA fibers. The precipitation of 


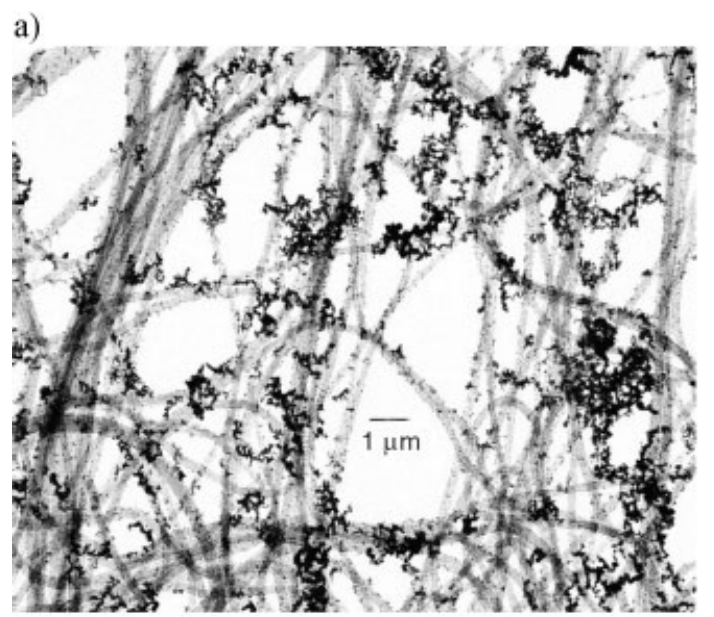

b)

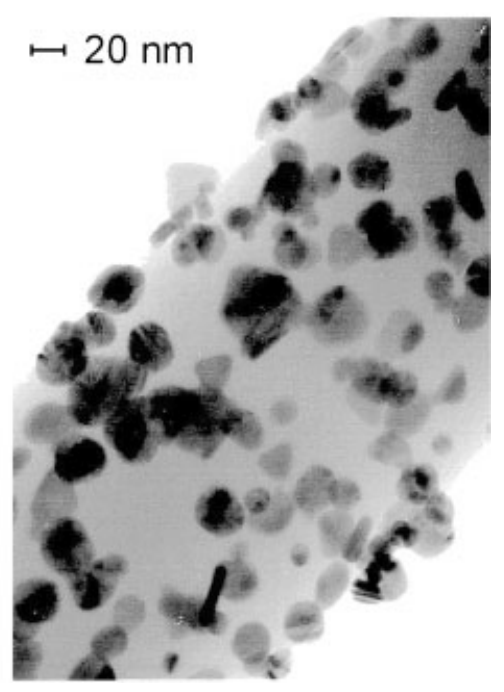

Figure 3. TEM images of silver nanoparticles nucleated on P(AN-GMA) fibers: a) low and b) high magnification.

silver atoms onto the fiber surface occurs in the same way. Unlike the first case, the atoms mainly grow by transfer of silver ions onto the particle surface. The lower the number of oxirane groups on the polymer backbone, the less silver atoms nucleate on the fibers surface. Thus, in short deposition times, the P(AN-GMA) fiber provides smaller particles as compared to PGMA. An additional systematic study to investigate the critical parameters such as GMA variation on the polymer backbone, degree of hydrazination, and concentration of silver nitrate in the surrounding solution, is needed to clarify the real mechanism of particle growth on P(AN-GMA) and PGMA.

The kinetics of crystalline particle growth follows the empirical equation

$$
d^{n}-d_{0}^{n}=k t
$$

where $d$ is the mean particle size, $d_{0}$ is the mean nuclei size or initial particle size, $k$ is temperature-dependent con-
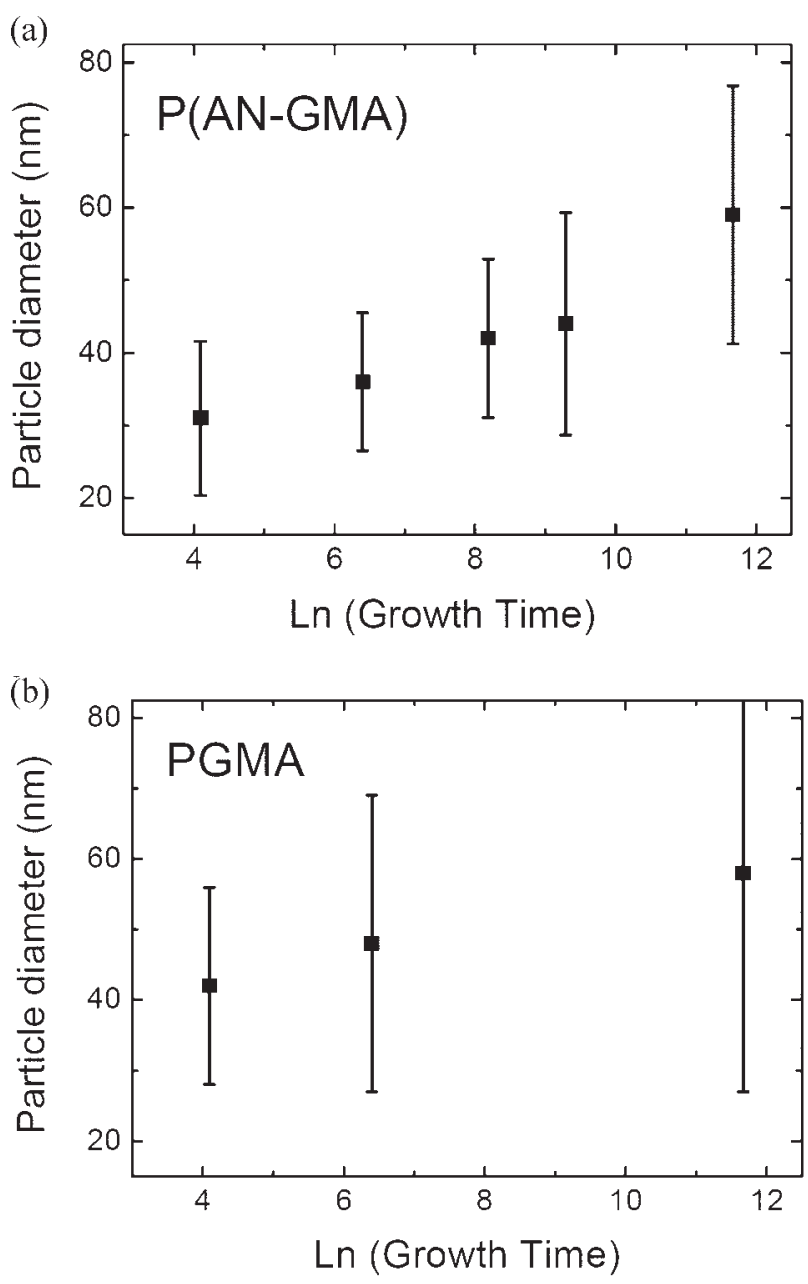

Figure 4. The particle mean diameters as a function of logarithm of deposition time in seconds on a) $\mathrm{P}(\mathrm{AN}-\mathrm{GMA})$ and $b$ ) PGMA.

stant, $t$ is time, and $n$ is the growth kinetic exponent, $d>d_{0}$. Assuming that the initial nucleus site (i.e., critical nucleus size) is much smaller than the particle size at $t=0$, this expression can be written as

$$
d^{n}=k_{0} \exp \left(\frac{-\underline{O}}{R T}\right) t
$$

where $k_{0}$ is a constant, $R$ is the gas constant, $T$ is the absolute temperature, and $Q$ is the activation energy for the mechanism that controls the growth. The value $n$, which provides important information about the growth kinetics of the particles, can be obtained by plotting ln $d$ versus $\ln t$ for a given temperature $T$. The plot of Figure 5 shows ln $d$ of particles produced on the two electrospun substrates as a function of $\ln t$. A linear behavior is found in both cases. The values of $n$ that were calculated from the slope of the best-fit lines were 6.6 for the particles nucleated on PGMA and 12.5 for the ones obtained on 


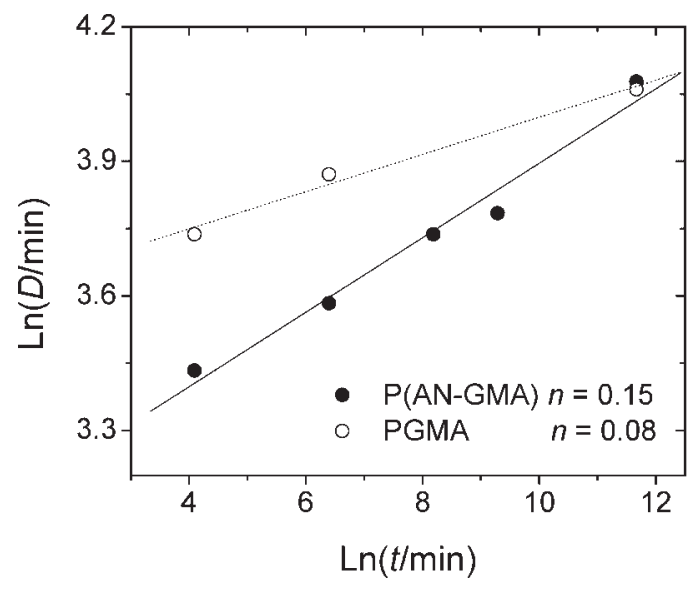

Figure 5. Plot of In $d$ of silver particles as a function of In $t$ for $\mathrm{P}(\mathrm{AN}-\mathrm{GMA})$ and PGMA fibers. The values of $n$ that were calculated from the slope of the best-fit lines were 6.6 for the particles nucleated on PGMA and 12.5 for the ones on P(AN-GMA).

$\mathrm{P}(\mathrm{AN}-\mathrm{GMA})$. These values suggest that the particle growth rate on PGMA is less than that on P(AN-GMA). Figure 6 provides cumulative frequency distributions of the particle diameters obtained from different deposition times. Increasing the coating time broadens the particle size distributions in both substrates. It appears that the particles produced on PGMA in $24 \mathrm{~h}$ show the broadest distribution.

Silver particles nucleate uniformly on the fiber surface. However, silver atoms do not only nucleate on the fiber surface but also in the solution. Figure 7 illustrates, along with silver particles on the electrospun fibers, silver agglomerates with fractal morphology in between the fibers. Nucleation events that take place on the fibers' surface are mediated by localized reduction with the help of bound hydrazine molecules (heterogeneous). However, some silver atoms appear to nucleate homogeneously in the solution. The latter causes uncontrolled crystal growth and agglomeration of individual silver particles that bundle the fibers together. The bundling of fibers is regarded as disadvantageous because it leads to an increase in the size of pores between the electrospun fibers and, therefore, decreases the surface area-to-volume ratio of both the silver particles and electrospun fibers. The actual mechanism of undesirable crystal growth between the fibers is not fully understood but it is speculated that the nucleation centers on two neighboring fibers which are in close proximity to each other adhere and crystal growth bridges the two fibers.

$\mathrm{P}(\mathrm{AN}-\mathrm{GMA})$ and $\mathrm{P}(\mathrm{AN}-\mathrm{GMA}) / \mathrm{Ag}$ mats were heated in oxidative media to remove the polymer and to determine the amount of metal on the electrospun mat. Thermoanalytical profiles of $\mathrm{P}(\mathrm{AN}-\mathrm{GMA})$ and Ag-coated $\mathrm{P}(\mathrm{AN}$ GMA) are presented in Figure 8. The thermooxidative decomposition of two products follows different paths
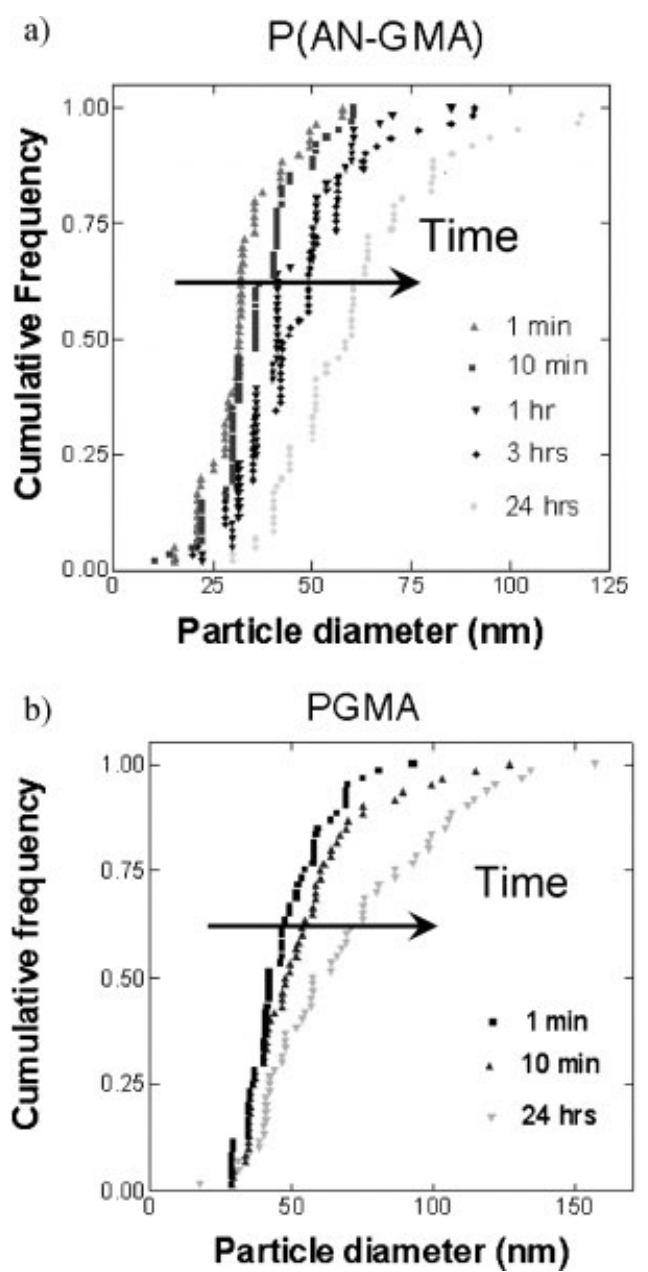

Figure 6. Cumulative distributions of particles on $\mathrm{P}(\mathrm{AN}-\mathrm{GMA})$ and PGMA fibers.

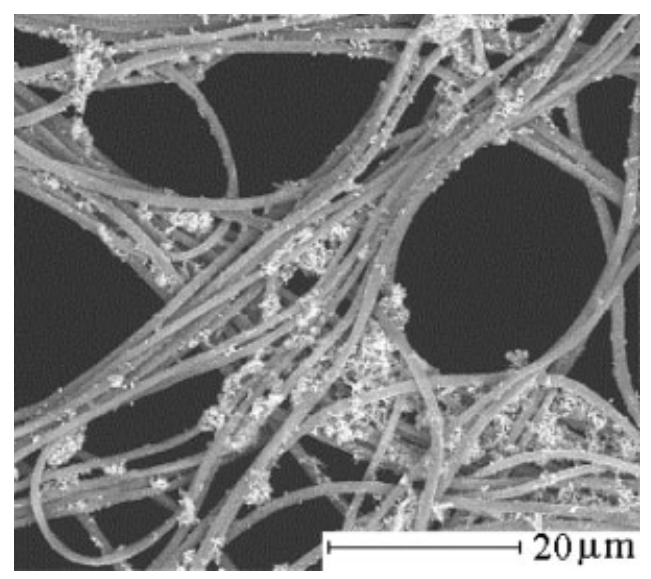

Figure 7. Bundling of electrospun PGMA fibers upon particle generation. 


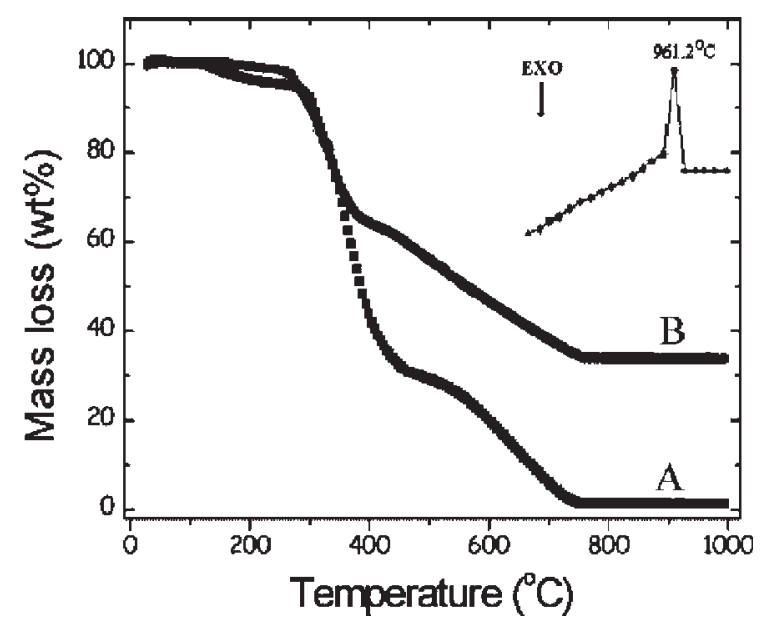

Figure 8. Thermo analytical curves of a) $\mathrm{P}(\mathrm{AN}-\mathrm{GMA})$ electrospun mats and b) Ag-coated P(AN-GMA) mat for 10 min. Mass loss difference in these two profiles suggests the amount of silver particles $\approx 35$ wt.-\% loaded onto electrospun fibers. The melting point of the $\mathrm{Ag}$ particles at $961^{\circ} \mathrm{C}$ is observed as an endothermic peak in the inset.

from room temperature to $1000^{\circ} \mathrm{C}$. Degradation occurs and mass decreases as the temperature increases. While only $2 \%$ of the polymer moiety remains beyond $770{ }^{\circ} \mathrm{C}$ under the thermooxidative environment for the electrospun mat of P(AN-GMA), 35 wt.-\% of the total mass was measured at the end of the thermal analysis for a metal-deposited sample. Mass loss of the samples before and after the metallization procedure was attributed to the amount of $\mathrm{Ag}$ deposited on the electrospun mat. The endothermic melting of Ag at $961^{\circ} \mathrm{C}$ is detected on the DTA trace. The mass percentage of the metallized electrospun mat consists of both metal particles produced on the nanofibers and particles formed between the nanofibers. The particles agglomerated between the fibers are unintentional and need to be minimized.

The catalytic activity of the silver nanoparticles generated on $\mathrm{P}(\mathrm{AN}-\mathrm{GMA})$ fibers was investigated by employing a reduction reaction that takes place between methylene blue dye and a reducing agent of $\mathrm{NaBH}_{4}$. This redox reaction does not occur when the reactants are brought together under ambient conditions unless there is a strong catalyst in the reaction medium. ${ }^{[10]}$ Therefore, this reaction was selected as a model system to acquire a qualitative understanding of the catalytic activity of the as-synthesized silver nanoparticles on the electrospun fibers. Methylene blue has a characteristic absorption band at $665 \mathrm{~nm}$. The intensity of the absorption at $665 \mathrm{~nm}$ is proportional to the concentration of methylene blue in the medium. The extent of the reaction can be followed by measuring the optical density of the reaction medium at $665 \mathrm{~nm}$. The surface plasmon of the silver particles, which is at $420 \mathrm{~nm}$, does not interfere with the band of
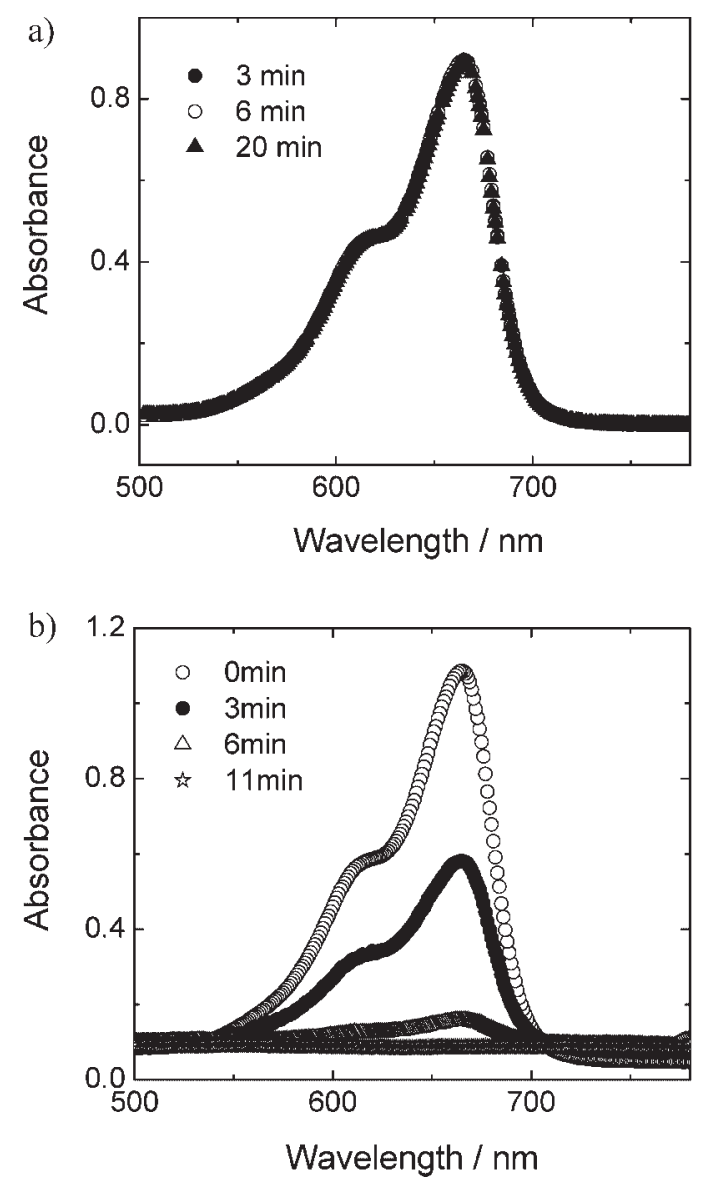

Figure 9. Visible spectra of a mixture of methylene blue dye and reducing agent $\mathrm{NaBH}_{4}$ a) in the absence and $b$ ) in the presence of catalytic silver particles on $\mathrm{P}(\mathrm{AN}-\mathrm{GMA})$ fibers.

methylene blue. Any optical activity taking place in the visible region originates from the catalytic performance of the silver particles in the redox reaction. Figure 9 presents the optical density of the reaction medium within $20 \mathrm{~min}$ of the reaction time. In the absence of silver particles (Figure $9(\mathrm{a}))$, the spectra of the reaction mixture $(3 \mathrm{~mL}$ of $2 \times 10^{-5} \mathrm{M}$ methylene blue and $1 \mathrm{~mL}$ of $0.1 \mathrm{M} \mathrm{NaBH}$ ) revealed a characteristic band of methylene blue at $665 \mathrm{~nm}$. No change in the spectra as a function of time indicates that the reduction reaction either did not occur at all or the extent of reaction is lower than the detection limit of optical spectroscopy. However, in the presence of $7 \mathrm{mg}$ of a P(AN-GMA) electrospun mat consisting of $20 \%$ silver nanoparticles, the absorption band at $665 \mathrm{~nm}$ disappears gradually. Figure 9 (b) illustrates the decrease in the optical density of the solution, which indicates the consumption of methylene blue in the reaction medium by the redox reaction. The reaction mixture turned from blue to colorless and the reaction was complete within $11 \mathrm{~min}$ in the presence of $\mathrm{Ag}$ particles. A detailed study on 
the catalytic activity of this particle/fibrous substrate system is under way. The arrangement of particles with respect to the electrospun fibers, i.e., whether they are at/ in/on the fibers, the size of the particles, the diameter of the fibers, and the amount of particles loaded into the fibers are the parameters needed to be examined.

\section{Conclusion}

We have shown the preparation of a surface active electrospun mat that consists of submicrometer diameter polymer fibers covered by monodisperse silver nanoparticles. The particles nucleate on the glycidyl methacrylate-based electrospun fibers. The glycidyl methacrylate residue content on the polymer backbone was the main parameter that controlled both particle size and particle growth. The particles generated on the electrospun fibers exhibited good catalytic activities, which were confirmed by the reduction of methylene blue dye using a reducing agent $\mathrm{NaBH}_{4}$. The present design of the nanocomposite materials may find various potential applications not only in catalysis but also in many fields where a large surface area of metal particles is utilized. A simple and inexpensive way was introduced for the preparation of this composite material, which employed electrospinning and electroless plating. From the stand point of industrial scale up production, the combination of these two solution processing techniques is promising for the production of nanoparticles associated with polymeric materials.

Acknowledgements: The authors thank Mr. A. Alkan from Brissa for electron microscope work and Dr. S. Özçelik for his helpful discussions.

Received: October 22, 2007; Revised: December 25, 2007; Accepted: January 4, 2008; DOI: 10.1002/macp.200700544

Keywords: catalysis; fibers; nanoparticles; particle nucleation; particle size distribution

[1] C. Burda, X. B. Chen, R. Narayanan, M. A. El-Sayed, Chem. Rev. 2005, 105, 1025.

[2] A. Haryono, W.H. Binder, Small 2006, 2, 600.

[3] L.M. Liz-Marzan, Langmuir 2006, 22, 32.

[4] Y.G. Sun, Y.N. Xia, Science 2002, 298, 2176.

[5] J. Chen, F. Saeki, B.J. Wiley, H. Cang, M.J. Cobb, Z.Y. Li, L. Au, H. Zhang, M.B. Kimmey, X.D. Li, Y. Xia, Nano Lett. 2005, 5, 473.

[6] A. Roucoux, J. Schulz, H. Patin, Chem. Rev. 2002, 102, 3757.
[7] A.N. Butenko, A.S. Savenkov, Russian J. Appl. Chem. 2000, 73, 1942.

[8] J.R. Monnier, J.W. Medlin, M.A. Barteau, J. Catal. 2001, 203, 362.

[9] W. Caseri, Macromol. Rapid Commun. 2000, 21, 705.

[10] A.C. Patel, S.X. Li, C. Wang, W.J. Zhang, Y. Wei, Chem. Mater 2007, 19, 1231.

[11] J.F. Ciebien, R.T. Clay, B.H. Sohn, R.E. Cohen, New J. Chem. 1998, 22, 685.

[12] R.F. Ziolo, E.P. Giannelis, B.A. Weinstein, M.P. Ohoro, B.N Ganguly, V. Mehrotra, M.W. Russell, D.R. Huffman, Science 1992, 257, 219.

[13] [13a] M.M. Demir, M.A. Gulgun, Y.Z. Menceloglu, B. Erman, S.S. Abramchuk, E.E. Makhaeva, A.R. Khokhlov, V.G. Matveeva, M.G. Sulman, Macromolecules 2004, 37, 1787; [13b]WO2005/021845 (2005) Sabanci University, invs.: M.M. Demir, M.A. Gulgun, Y.Z. Menceloglu,

[14] H.Y. Wang, X.F. Lu, Y.Y. Zhao, C. Wang, Mater. Lett. 2006, 60 2480.

[15] M.K. Abyaneh, D. Paramanik, S. Varma, S.W. Gosavi, S.K Kulkarni, J. Phys. D: Appl. Phys. 2007, 40, 3771.

[16] O..B. Yang, D.M. Li, Y.L. Hong, Z.Y. Li, C. Wang, S.L. Oui, Y. Wei, Synth. Met. 2003, 137, 973.

[17] N.J. Pinto, P. Carrion, J.X. Quinones, Mater. Sci. Eng. A 2004, 366, 1-5.

[18] M.R. Bockstaller, R.A. Mickiewicz, E.L. Thomas, Adv. Mater 2005, 17, 1331.

[19] A. Jain, U. Wiesner, Macromolecules 2004, 37, 5665.

[20] A. Greiner, J.H. Wendorff, Angew. Chem. 2007, 46, 5670

[21] S.A. Theron, E. Zussman, A.L. Yarin, Polymer 2004, 45, 2017.

[22] S.V. Fridrikh, J.H. Yu, M.P. Brenner, G.C. Rutledge, Phys. Rev. Lett. 2003, 90, 144502.

[23] X.Y. Xu, O.B. Yang, Y.Z. Wang, H.J. Yu, X.S. Chen, X.B. Jing, Eur. Polym. J. 2006, 42, 2081.

[24] K.H. Hong, J.L. Park, I.H. Sul, J.H. Youk, T.J. Kang, J. Polym. Sci., Part B: Polym. Phys. 2006, 44, 2468.

[25] K.H. Hong, Polym. Eng. Sci. 2007, 47, 43.

[26] M. Jin, X.T. Zhang, S. Nishimoto, Z.Y. Liu, D.A. Tryk, T Murakami, A. Fujishima, Nanotechnology 2007, 18.

[27] W.K. Son, J.H. Youk, W.H. Park, Carbohydr. Polym. 2006, 65 430.

[28] N. Bicak, S. Sungur, N. Tan, F. Bensebaa, Y. Deslandes, J. Polym. Sci., Part A: Polym. Chem. 2002, 40, 748.

[29] M.Y. Arica, G. Bayramoglu, Biochem. Eng. J. 2004, 20, 73.

[30] O. Burtovyy, V. Klep, H.C. Chen, R.K. Hu, C.C. Lin, I. Luzinov, J. Macromol. Sci., Part B: Phys. 2007, 46, 137.

[31] R. Krishnan, K.S.V. Srinivasan, Macromolecules 2003, 36, 1769.

[32] H.H. Lee, K.S. Chou, K.C. Huang, Nanotechnology 2005, 16 2436.

[33] S. Serghinimonim, P.R. Norton, R.J. Puddephatt, J. Phys. Chem. B 1997, 101, 7808.

[34] Y.K. Mishra, S. Mohapatra, D. Kabiraj, B. Mohanta, N.P. Lalla, J.C. Pivin, D.K. Avasthi, Scripta Materialia 2007, 56, 629.

[35] N. Koura, in: "Electroless Plating: Fundamentals, Applications" G.O. Mallory, Ed., American Electroplaters, Surface Finishers Society Orlando 1990.

[36] M.M. Demir, I. Yilgor, E. Yilgor, B. Erman, Polymer 2002, 43 , 3303.

[37] B. Smith, "Infrared Spectral Interpretation: A Aystematic Approach" CRC Press London 1999. 